\title{
Understanding Streaming in Dictyostelium discoideum: Theory versus Experiments
}

\author{
J. C. Dallon \\ dallon@math.byu.edu \\ Brittany Dalton \\ Chelsea Malani
}

Follow this and additional works at: https://scholarsarchive.byu.edu/facpub

Part of the Mathematics Commons

Original Publication Citation

Bulletin of Mathematical Biology 73(7)

\section{BYU ScholarsArchive Citation}

Dallon, J. C.; Dalton, Brittany; and Malani, Chelsea, "Understanding Streaming in Dictyostelium discoideum: Theory versus Experiments" (2011). Faculty Publications. 2714.

https://scholarsarchive.byu.edu/facpub/2714 
Bulletin of Mathematical Biology (2010) 01, 1-25

\title{
Understanding Streaming in Dictyostelium discoideum: Theory versus Experiments
}

\author{
J. C. DALLON, BRITTANY DALTON, AND CHELSEA MALANI \\ Department of Mathematics, Brigham Young University, TMCB 312, Provo, UT \\ 84602-6539
}

June 28, 2010

Recent experimental work involving Dictyostelium discoideum seems to contradict several theoretical models. Experiments suggest that localization of the release of the chemoattractant cyclic adenosine monophosphate to the uropod of the cell is important for stream formation during aggregation. Yet several mathematical models are able to reproduce streaming as the cells aggregate without taking into account localization of the chemoattractant. A careful analysis of the experiments and the theory suggests the two major features of the system which are important to stream formation are random cell motion and chemotaxis to regions of higher cell density. Random cell motion acts to reduce streaming, whereas chemotaxis to regions of higher cell density reinforces streaming. With this understanding, the experimental results can be explained in a manner consistent with the theoretical results. In all the experiments, alterations in the two main factors of random motion and chemotaxis to regions of higher cell density, not the localization of the release of the chemoattractant, can explain the results as they relate to streaming. Additionally, a comparison of results from a mathematical model that simulates cells which localize the chemoattractant and cells which do not shows little difference in the streaming patterns.

(c) 2010 Society for Mathematical Biology

Key words: aggregation, cell movement, orientation, mathematical models, localization

\section{Introduction}

Dictyostelium discoideum (Dd) is a cellular slime mold commonly studied to better understand chemotaxis and cellular development (for review, see (Loomis, 1975; Kessin, 2001)). Under normal circumstances these amoeba live individually feeding on bacteria. After a period of starvation they undergo developmental changes, including becoming chemotactically sensitive to cyclic adenosine monophosphate (cAMP) and also being able to produce cAMP when stimulated by external cAMP. Seemingly random cells periodically emit a pulse of cAMP which is relayed by other cells causing aggregations to form at the pulsing cells. Once the cells have aggregated into slugs, they form fruiting bodies consisting of stalk cells and spore cells. Several mathematical models have been developed to understand the mechanisms underlying aggregation (Parnas \& Segel, 1977; Levine et al., 1996; Vasiev et al., 1994; MacKay, 1978; Dallon \& Othmer, 1997; Palsson \& Othmer, 2000; Höfer et al., 1995; Savill \& Hogeweg, 1997). These models successfully mimic the aggregation of a field of cells into aggregation streams and the condensation

0092-8240/10/010001 + $25 \$ \$ 17.00 / 0 \quad$ mb10???? C 2010 Society for Mathematical Biology 
of the streams into a group. The modeling varies greatly in the methods used, which have included discrete formulations (MacKay, 1978; Dallon \& Othmer, 1997; Palsson \& Othmer, 2000), continuum formulations (Höfer et al., 1995), cellular automata and energy minimization techniques (Savill \& Hogeweg, 1997), and ideas from fluid dynamics (Vasiev et al., 1997).

Recent experimentation has suggested that localization of adenylyl cyclase ACA (ACA), one of the enzymes that produces cAMP, at the posterior of the cell is necessary for stream formation in Dd (Kriebel et al., 2003; Kriebel et al., 2008), yet theoretical models exhibit streaming phenomena without incorporation of localization. In this manuscript we explore the effects of localizing the output of cAMP. We assume that localizing ACA also localizes the output of cAMP. Our main goal is to clarify the apparent contradiction between the theoretical modeling and the experimental work. To do this, we pose two questions which we will attempt to answer. First, what is streaming? Second, why do theoretical models capture streaming behavior without localization of cAMP? Before considering those questions we first review the major experiments which focus on the disruption of streaming.

Kriebel and coworkers reported the results of a set of experiments which led them to the conclusion that localization of cAMP was necessary for stream formation (Kriebel et al., 2003). In subsequent work which focused on the subcellular processes necessary for the cAMP localization (Kriebel et al., 2008) more experiments were performed which are relevant to our discussion. Additionally, Hilgardt and coworkers observed that isopropylidenadenosin (IPA) causes Dd to aggregate in a streamless manner (Hilgardt et al., 2008). We briefly summarize those experiments here and will refer to them throughout the rest of the paper. Initially Kriebel and colleagues showed that cells lacking ACA, $a c a^{-}$do not stream when stimulated by a spatial cAMP gradient (generated by a pipette) whereas wild type cells do stream. To further investigate this they developed a method to visualize the ACA distribution within a cell by fusing ACA with yellow florescent protein (YFP) and transforming different cell types with the ACA-YFP expression plasmid. They transformed $a c a^{-}$to form ACA-YFP/aca- and ACA-C-YFP/aca- where the ACA-C is a mutant form of ACA that is constituitively active. When stimulated by a pipette $\mathrm{ACA}-\mathrm{YFP} / a c a^{-}$cells stream but $\mathrm{ACA}-\mathrm{C}-\mathrm{YFP} / a c a^{-}$cells do not. The ACA is localized at the uropod for ACA-YFP $/ a c a^{-}$cells but it is not for ACA-C-YFP/aca cells. They also found interesting results as they observed individual cells for mixed populations of cell types. Based on ACA-C-YFP/aca experiments and the experiments with mixed populations of cells they concluded that localization of ACA is necessary for streaming. They further observed that $\mathrm{myoII}^{-}$cells, cells which lack myosin II, aggregate but do not stream when stimulated with a spatial gradient of cAMP. We do not address the results of the experiments observing individual cells in the streaming process because our models consider streaming at a macroscopic scale and not at the individual cell-cell adhesion level (see below for more details). In the follow up work by Kriebel and coworkers, they found that $\mathrm{ACA}-\mathrm{YFP} / a c a^{-}$cells treated with Nocodazole a drug which disrupts the microtubule network or cycloheximide (CHX) a drug which inhibits protein synthesis do not stream. The two mutants $c h c^{-}$and $c l c^{-}$lacking clathrin ag- 
gregated when stimulated by a pipette but did not exhibit streaming. Finally, Hilgardt and coworkers found that treating wild type cells with IPA caused different aggregation patterns. In these experiments the cells were not stimulated to aggregate by a pipette but aggregated due to naturally occurring pacemaking regions. The cells treated with IPA which affects the binding of cAMP to the membrane bound receptors, aggregated but did not form streaming patterns. We now return to the two questions posed earlier, what is streaming and why do theoretical models capture streaming behavior without localization of cAMP?

What is streaming? At a macroscopic scale, the cell density pattern forms vein-like or branch-like formations which move toward the aggregation center. If the aggregation center is a point at the origin and we consider polar coordinates, then uniform aggregation would be described as a density function which over time increases as the radius decreases but does not vary with respect to the angle. Aggregation that involved streaming would require that the density varied with respect to both variables, angle and radius. What feature of the system causes the cell density to vary with respect to variables other than distance from the aggregation center? Analysis from Dallon \& Othmer (Dallon \& Othmer, 1997) indicates that the break up into streams is reinforced and stabilized by the ability of cells to relay the cAMP signal. That is, once there is a variation in the density of the cells in some variable other than distance from the aggregation center the relay ability of the cells will act as a positive feedback reinforcing and enhancing the density difference. Since there is a higher density of cells in one region, when those cells relay the cAMP signal, it will be stronger than in the low density regions, thus recruiting more cells. This motivates the definition of streaming which we present. In order to be clear, we define streaming for the purposes of this paper in a narrow way. If when cells aggregate to a source, they do so by first moving in a direction toward an area which has a locally higher cell density and then eventually move to the source they are said to be streaming.

Our definition is made to capture what appears to be streaming at the macroscopic scale and not the cellular scale. Although this is not the case in Dd, one can imagine that at a macroscopic level cells may appear to be streaming into the center, but at the cellular level there is no cell-cell contact. At the cellular scale it may not appear to be a stream of cells. We do not address any of the observed behavior of Dd cells in terms of cell-cell contact with streaming, the role of trails of membranous structures left behind as the cell migrates (Kriebel et al., 2008), or the possibility of regions of differential adhesion on the cell membrane.

The second question posed is why do theoretical models capture streaming behavior without subcellular localization of the chemoattractant source? We have already alluded to the answer in a previous paragraph when we defined streaming. To be more precise and thorough, we begin by briefly discussing four models which show streaming behavior as defined above. The four models which we refer to are the MacKay model (MacKay, 1978), the Dallon \& Othmer model (Dallon \& Othmer, 1997), the Hoefer et. al. model (Hoefer et al., 1995; Hofer \& Maini, 1997), and the Vasiev et. al. model (Vasiev et al., 1997). None of these models explicitly consider localization of cAMP, but all show 
streaming.

The MacKay model of Dd aggregation assumes the cells are discrete points which move in the plane and does not allow for localization of the cAMP output. The model is formulated as an algorithm with the cells following certain rules such as the cells move when the cAMP concentration is above threshold and they move in the direction of the maximum spatial gradient. The cells relay the cAMP signal by outputting a fixed amount of cAMP when they begin to move and the cAMP diffuses. With this simple system MacKay was able to reproduce aggregation patterns that streamed (MacKay, 1978).

The Dallon \& Othmer model uses the same formulation as the MacKay model but treats the diffusion and the signal transduction of cAMP in a more sophisticated manner. It assumes that cells are discrete points free to move in the plane. They respond to the cAMP concentration gradient which is modeled as a continuum variable which diffuses. The cells act as point sources or sinks of cAMP, depending on the state of the cell, and move up the chemical gradient. The transduction of the extracellular cAMP signal into the intracellular signal is based on the Tang \& Othmer model (Tang \& Othmer, 1995) which involves solving several coupled ordinary differential equations for each cell. This model reproduces several features of the biological system including streaming patterns. Since the cells are treated as point sources or sinks, localization of the cAMP output is not possible. The signal transduction model assumes that the inhibition of the production of cAMP occurs due to an inactivation of the receptor which stimulates the production. This is now known to be incorrect, since the competitive interference does not take place at the level of the receptors. Yet the input-output response of the cells compares well with experiments and the error would likely not affect the streaming behavior. It is a variation of this model which we use to investigate the effect of localization of ACA on streaming in this manuscript.

The Hoefer et. al. model (Hoefer et al., 1995; Hofer \& Maini, 1997) assumes the cell density to be a continuum variable which is governed by a conservation equation regarding the cell flux. This gives a partial differential equation which the density variable satisfies. The cAMP dynamics are given by two coupled differential equations which are reaction diffusion equations. Again in this model formulation since the cells are not represented individually, there is no possibility to localize the cAMP production. The production is a function of the cell density variable. This model also captures the streaming behavior of the system.

Finally, the model developed by Vasiev et. al. (Vasiev et al., 1997) assumes that the cell density is a continuum but that it behaves as either a viscous liquid or a non viscous liquid and the Navier-Stokes equations are used to model the cell density. The cAMP dynamics are modeled using the FitzHugh-Nagumo reaction diffusion equations. This model also reproduces streaming behavior.

What assumptions are made in these four models which are relevant to streaming and localization of ACA? The specifics of the cAMP signal transduction do not seem to be important. In MacKay the cAMP signal is simply imposed, in Dallon \& Othmer a detailed transduction model is used with some incorrect hypothesis but the input- 
output responses are realistic, and in the other two models mathematical caricatures are used to simulate the cAMP signal, yet they all produce streaming. Experiments with $a c a^{-}$cells, cells which lack ACA, aggregate in a spatial gradient but do not give rise to streams (Kriebel et al., 2003). This supports the idea that the relay of cAMP is important. Common to all four models is that the local concentration of cAMP depends on the local cell density, i.e. the cells relay the signal. The other common factor which seems essential is that cells move up the chemical gradient. This allows the small initial variations in density to be reinforced as the cells move up gradients of cAMP which gives rise to streams of cells. Another assumption in all the models is that the cell bodies do not obstruct the cAMP diffusion. This is clearly not realistic and could significantly alter the cAMP gradient calculated in the models. Finally, the models assume cells have either no volume (MacKay, 1978; Dallon \& Othmer, 1997) or the volume is averaged over space to give a continuum variable for the density (Hoefer et al., 1995; Vasiev et al., 1997). For the remainder of the paper we will explore how these assumptions affect streaming and relate to the experiments. First we will give a simple mathematical analysis of diffusion and show how moving the source 5 to 20 microns affects the chemical profile. To understand how the cell body affects diffusion of the cAMP signal, we will give results from two dimensional simulations where the cells do not move but block the diffusion of cAMP (Dallon \& Othmer, 1998). Next we will modify the Dallon \& Othmer model by adding mutant cells and stimulation with a pipette to better mimic the experimental data. We will then change the scale of the model and finally we will be in a position to localize the cAMP signal to the posterior region of the cell. We conclude with a discussion.

\section{Analysis of simple diffusion}

The first step to understanding how localizing the production of cAMP to one region of a cell affects streaming is to determine how it affects the concentration gradient. A simple mathematical calculation of how a point source diffuses on a line gives some insight. The differences in the computer simulations, with cells which output cAMP uniformly around the membrane or output it at the cell center, and a cell which localizes the signal at the uropod would be less than 7 percent for a cell thirty microns away at five seconds after relay and less than 2 percent 20 seconds after relay and the absolute concentrations are very small. We assumed that the cells outputs $1 \times 10^{7}$ molecules, a typical amount which a cell will output when stimulated (Roos et al., 1975; Gerisch \& Wick, 1975), and we use $15000 \mu^{2} / \min$ (Cohen et al., 1975) for the diffusion coefficient of cAMP. It thus seems unlikely that the assumption that the cells output the cAMP at their center or uniformly and not at the uropod should change the streaming patterns which are seen in the simulations. The main problem with this analysis is that, in reality, the cell body blocks the diffusion of the cAMP. In the next section we will consider how the cell body affects the diffusion profile. 


\section{Modelling diffusion around the cell body}

Using a previously developed model of the chemotactic signal near a cell membrane (Dallon \& Othmer, 1998), we determined the difference in the signal of a cell which produces the cAMP on only one side compared to the signal of a cell which produces the cAMP uniformly around the cell membrane. The model assumes that there are two cylindrical cells having radius $r_{0}=5.5$ microns. To reduce the model to a 2 dimensional model the concentrations are assumed to be uniform in the vertical direction, and cAMP is allowed to diffuse in the region exterior to the cells. The equation for the extracellular cAMP, $w_{5}$ is

$$
\frac{\partial w_{5}}{\partial \tau}=D_{1} \nabla^{2} w_{5}
$$

exterior to the cells, with boundary conditions

$$
-D_{1} \hat{\mathbf{n}}_{\mathbf{o}}{ }^{1} \cdot \nabla w_{5}=-\gamma_{7} \frac{w_{5}}{w_{5}+\gamma_{6}}
$$

on the boundary of the receiver and

$$
-D_{1} \hat{\mathbf{n}}_{\mathbf{o}}^{2} \cdot \nabla w_{5}=\frac{V_{c}}{k_{5}[i P D E]_{T} A_{c} r_{0}} K F(t)-H(t-2)\left(\gamma_{7} \frac{w_{5}}{w_{5}+\gamma_{6}}\right)
$$

on the boundary of the signaling cell. Here $\hat{\mathbf{n}}_{\mathbf{o}}^{i}$ is the outward normal to the cell boundary, $K$ converts molecules per cell to micromoles, $D_{1}$ corresponds to the dimensionless diffusion coefficient when the dimensional diffusion coefficient is $15000 \mu^{2} / \mathrm{min}, k_{5}=3.75 / \mathrm{sec}$, $\gamma_{7}=36.7, \gamma_{6}=0.29,[I P D E]=1.2 \times 10^{-8}$ micromoles $/$ cell, $\mathrm{h}=2$ microns, $V_{c}=\pi r_{0}^{2} h$ is the volume of the cell, and $A_{c}=2 \pi r_{0} h$ is the area of the cell. In the simulations shown the signaling cell outputs cAMP in a piecewise linear function which satisfies

$$
F(t)= \begin{cases}F_{m} t & 0 \leq t<1 \\ F_{m}(2-t) & 1 \leq t \leq 2 \\ 0 & \text { otherwise }\end{cases}
$$

where $t$ is in minutes and $F_{m}$ is set at $1 \times 10^{7}$ molecules per cell per minute squared. The function $F(t)$ takes into account not only the release of cAMP by the signaling cell, but also enzymatic degradation by mPDE on the cell surface. For simulations where the ACA is localized and thus the production of cAMP is only at the back end of the cell, the function $F(t)$ depends also upon the location on the membrane. It is zero for the front half of the membrane and two times equation 4 on the back half. This makes the signaling cell in either simulation output the same amount of cAMP, which is a total of $1 \times 10^{7}$ molecules per cell per pulse. Otherwise the two simulations are the same. 
2.1. Results. In both simulations there was a signaling cell on the left and another cell on the right (see figure 1). The signaling cell started to release cAMP at the beginning of the simulation and continued to release it for two minutes as described above. The simulation where the signaling cell released cAMP uniformly along the membrane had concentration contours which would be concentric circles if not for the second cell. Instead they were compressed between the two cells due to the second cell blocking the diffusion (see figure 1a). Comparing the concentration along the line through the center of the two cells for a simulation with cAMP uniformly released along the membrane and a simulation where cAMP is released only on the left half of the cell membrane (see figure $1 \mathrm{~b}$ and $\mathrm{c}$ ) at 12 seconds shows that the concentration profile near the receiving cell is very similar. The concentrations never vary more than 0.0015 micromolars and the relative difference (uniform minus localized all divided by uniform) at 12 seconds has a maximum of about 10 percent. The results were similar to the analysis done in the previous section. Although the cell body does have an effect on the concentration profiles it does not seem to significantly alter the conclusion drawn previously. That is, even when the cell body is taken into account the concentration a receiving cell senses is not much different whether the signaling cell localizes the release or not. The fact that the theoretical models allow the cAMP to freely diffuse despite the obstructions due to the cell bodies does not seem to be a significant simplification when considering streaming. Now that we have this understanding we will continue the investigation by modifying the Dallon \& Othmer model but retaining the simplification of freely diffusing cAMP in the plane.

\section{The basic aggregation model}

The mathematical model for aggregation of Dd is a modification of the Dallon \& Othmer model (Dallon \& Othmer, 1997) described in previous work (Dallon et al., 2006). We will briefly review the main framework of the model. The Dd cells are assumed to be discrete cells which respond to the cAMP concentration by modifying it and moving. They modify the cAMP concentration by producing and degrading cAMP depending upon the local external cAMP concentration. The cells move up spatial gradients of cAMP concentration. Although originally the cells were treated as points in space (Dallon \& Othmer, 1997), in this version the cells have volume, cannot occupy the same space, and adhere to one another (Dallon et al., 2006).

Depending on the simulations the cells modify the cAMP concentration using either the Tang \& Othmer cAMP transduction model (Tang \& Othmer, 1995) (TO) or the simple transduction model. In order to mimic behavior of various mutants we developed and used a simple transduction model. As was mentioned earlier, very different models of cAMP give streaming patterns indicating that the specific details of the transduction model are less important when considering streaming. The TO model was designed to replicate the input output behavior of wild type cells over a large range of inputs. We were not able to modify it easily to mimic the behavior of the relevant mutants. Thus, we developed a simple model which was easily modified to mimic the mutants. We compared 


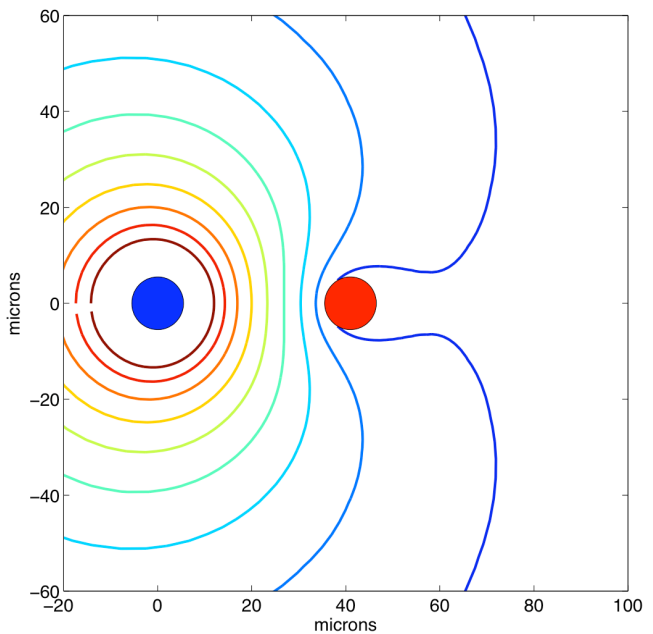

a

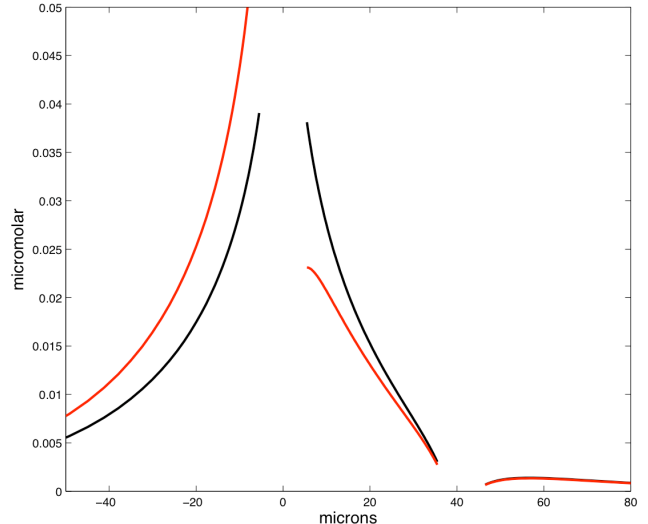

$\mathrm{b}$

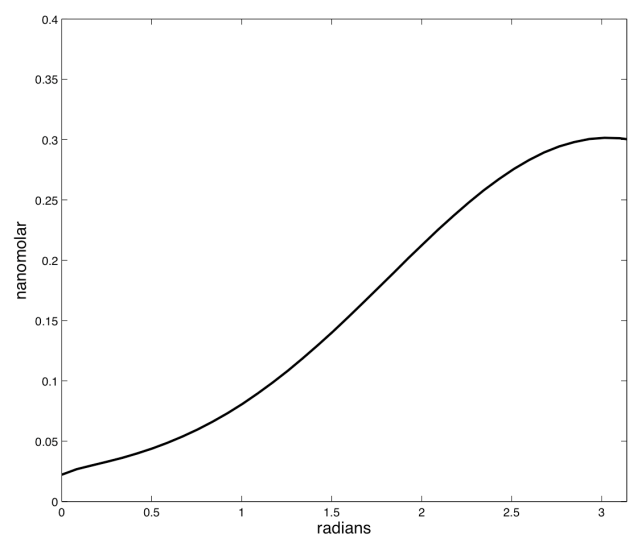

$\mathrm{C}$

Figure 1: The concentration of cAMP is shown in simulations where the cell body blocks diffusion. The concentration contours are shown in a) for the typical setup where the left cell releases cAMP uniformly around its membrane in a two minute interval. The contours are shown at 36 seconds after the cell starts releasing cAMP. The contours are for $0.01,0.02,0.03,0.04,0.05,0.06,0.07$, 0.08, and 0.09 micromolar going from the ones closest to the right cell to the ones closest to the left cell respectively. In b) the concentration on the line through the two cell centers is shown for a simulation where the release is localized to the left side of the left cell (red) and for a simulation where the release is uniform over the cell membrane (black) at 12 seconds. In c) the difference (the uniform simulations minus the localized simulation) in concentration at the non-signaling (right) cell's membrane is shown at 12 seconds after signaling starts. The simulation is symmetric with respect to the line $y=0$ so the concentration is shown for cell membrane in the region of the plane $y>0$ (the top half of the cell membrane). The zero angle is the direction pointing away from the signaling cell. 
all results when possible with the aggregation model using the TO dynamics since we assumed it gives the more accurate cAMP concentrations for wild type cells.

The TO cAMP transduction model is given by the following equations.

$$
\begin{aligned}
& \frac{d w_{1}^{i}}{d \tau}=\alpha_{4} u_{2}^{i}-w_{1}^{i}-\alpha_{4} u_{2}^{i} w_{1}^{i} \\
& \frac{d w_{2}^{i}}{d \tau}=\beta_{2} \beta_{3} c_{2} u_{4}^{i}-\beta_{5} w_{2}^{i}+\beta_{6} c_{3} w_{3}^{i}-c_{3} \beta_{4} u_{1}^{i} w_{2}^{i}-\beta_{2} \beta_{3} c_{2} u_{4}^{i}\left(w_{2}^{i}+c_{3} w_{3}^{i}\right) \\
& \frac{d w_{3}^{i}}{d \tau}=-\left(\beta_{5}+\beta_{6}\right) w_{3}^{i}+\beta_{4} u_{1}^{i} w_{2}^{i} \\
& \frac{d w_{4}^{i}}{d \tau}=\gamma_{1} \gamma_{2} w_{1}^{i}+\Gamma_{5}\left(1-\Gamma_{7} w_{1}^{i}\right)-\gamma_{4} \frac{w_{4}^{i}}{w_{4}^{i}+\gamma_{3}}-\operatorname{sr}\left(w_{4}^{i}\right)
\end{aligned}
$$

where

$u_{1}^{i}=\frac{\alpha_{0} w_{5}\left(\mathbf{x}_{i}\right)+\left(\beta_{5}-\alpha_{0} w_{5}\left(\mathbf{x}_{i}\right)\right) w_{3}^{i}}{\alpha_{1}+\alpha_{0} w_{5}\left(\mathbf{x}_{i}\right)+\beta_{4} w_{2}^{i}} \quad u_{2}^{i}=\frac{\alpha_{2} \alpha_{3} c_{1} u_{1}^{i}\left(1-w_{1}^{i}\right)}{1+\alpha_{4}+\alpha_{2} \alpha_{3} c_{1} u_{1}^{i}-\alpha_{4} w_{1}^{i}} \quad u_{4}^{i}=\frac{\beta_{0} w_{5}\left(\mathbf{x}_{i}\right)}{\beta_{1}+\beta_{0} w_{5}\left(\mathbf{x}_{i}\right)}$.

Here superscript $i$ designates the $i^{t h}$ cell, whose position in the plane is denoted $\mathbf{x}_{i}$, and $s r$ is the function denoting the dimensionless secretion rate of cAMP. The parameter values used are given in Table 1 and are the same as those used in (Dallon et al., 2006) unless otherwise stated. The variables represent intracellular chemical complexes for the signal transduction pathway in a scheme which proposes a stimulatory pathway and a slower inhibitory pathway both using $\mathrm{G}$ proteins. Thus cAMP binds to the stimulatory receptor to form the complex represented by $u_{1}$, which causes the $\mathrm{G}$ protein to decouple and as a result frees the activated subunit, $u_{2}$. The activated subunit joins with adenylyl cyclase forming a complex represented by $w_{1}$ which catalyses the production of intracellular cAMP denoted $w_{4}$. Meanwhile, in the inhibitory pathway, cAMP binds to a receptor to form a complex represented by $u_{4}$ which also causes the production of an activated subunit of an inhibitory $\mathrm{G}$ protein denoted $w_{2}$. This inhibitory subunit binds with $u_{1}$, the bound stimulatory receptor, forming $w_{3}$ and stopping the stimulatory pathway. It is now known that the competitive interference does not take place at the level of receptors as hypothesized in the Tang \& Othmer model (Othmer \& Schaap, 1998). Still, the model is very useful as it reproduces the excitation, adaptation, and relay quantitatively correctly.

The evolution of extracellular cAMP, represented by $w_{5}$, is governed by the partial differential equation

$$
\begin{aligned}
\frac{\partial w_{5}(\mathbf{x})}{\partial \tau}= & \Delta_{1} \nabla^{2} w_{5}(\mathbf{x})-\hat{\gamma_{9}} \frac{w_{5}(\mathbf{x})}{w_{5}(\mathbf{x})+\gamma_{8}} \\
& +\sum_{i=1}^{N} \frac{k V_{c}}{V_{o}} \delta\left(\mathbf{x}_{i}-\mathbf{x}\right)\left(\operatorname{sr}\left(w_{4}^{i}\right)-\gamma_{7} \frac{w_{5}(\mathbf{x})}{w_{5}(\mathbf{x})+\gamma_{6}}\right) .
\end{aligned}
$$


For computational convenience we stack several cells at one location and thus simply multiply the cell contribution by a weight factor $k$. Here $\hat{\gamma_{9}}=\gamma_{9} \frac{N k V_{c}}{V_{o}}, N k$ is the number of cells, $V_{c}=696.9 \mu \mathrm{m}^{3}$ is the volume of a cell (which corresponds to a spherical cell with radius of about $5.5 \mu \mathrm{m}), V_{o}=1.375 \times 10^{8} \mu \mathrm{m}^{3}$ is the volume of the extracellular medium (assuming it to be $0.5 \mathrm{~cm}$ square and about $5.5 \mu \mathrm{m}$ thick), $\mathrm{x}$ is a generic point in the plane, and $\delta$ is the Dirac distribution. The terms on the right-hand side of the partial differential equation represent, in order, a diffusive contribution, the degradation due to external phosphodiesterase (PDE), which is assumed to be constant in space and time unless otherwise stated, the secretion of cAMP, and the degradation of cAMP by membrane-bound PDE. The cAMP signal is solved numerically as described elsewhere (Dallon, 2000), and with the exception of the cell motion, the algorithms are the same.

Table 1: Parameter Values. See (Tang \& Othmer, 1995) for a full description of dimensionless parameters.

\begin{tabular}{|c|c|c|c|c|}
\hline$\alpha_{0}=312.0$ & $\beta_{0}=61.0$ & $\gamma_{1}=323.2$ & $\gamma_{6}=11.6$ & $\Delta_{1}=4.0 \times 10^{-5}$ \\
\hline$\alpha_{1}=0.8$ & $\beta_{1}=16.0$ & $\gamma_{2}=$ varied & $\gamma_{7}=36.7$ & $s r_{1}=0.02$ \\
\hline$\alpha_{2}=2.67$ & $\beta_{2}=0.48$ & $\gamma_{3}=57.7$ & $\Gamma_{7}=1.09$ & $s r_{2}=0.65$ \\
\hline$\alpha_{3}=1.0$ & $\beta_{3}=1.0$ & $\gamma_{4}=350.0$ & $\gamma_{8}=750.0$ & $s w=0.5$ \\
\hline$\alpha_{4}=26.7$ & $\beta_{4}=2.0 \times 10^{4}$ & $\Gamma_{5}=2.4$ & $\gamma_{9}=959.3$ & $c_{1}=1.0$ \\
\hline$k_{2}=14750 \mathrm{nN} / \mathrm{mm}$ & $\beta_{5}=0.4$ & $\mu_{\text {cell }}=4250 \mathrm{nN} \mathrm{min} / \mathrm{mm}$ & $\mu_{s}=2250 \mathrm{nN} \mathrm{min} / \mathrm{mm}$ & $c_{2}=1.0$ \\
\hline$k_{a}=83800 \mathrm{nN} / \mathrm{mm}$ & $\beta_{6}=204.0$ & $\mu_{f}=425 \mathrm{nN} \mathrm{min} / \mathrm{mm}$ & $\mu_{a}=63000 \mathrm{nN} \mathrm{min} / \mathrm{mm}$ & $c_{3}=0.668$ \\
\hline
\end{tabular}

The simple transduction model is a caricature of cAMP dynamics. Wild type cells in the simple model, when stimulated output $5 \times 10^{6}$ molecules of cAMP in one minute (Roos et al., 1975; Gerisch \& Wick, 1975) and produce a basal level of cAMP which is $\frac{1}{120}$ times the stimulated output. After outputting the cAMP the cells are refractory for one minute, meaning they cannot be stimulated to output more cAMP (other than at a basal level). The basal level is based on the measurements that the peak production rate of stimulated wild type cells can be from 6 to 100 times the basal rate of production (Parent \& Devreotes, 1996; Devreotes \& Steck, 1979). The cells are stimulated to produce cAMP when the time derivative of the cAMP concentration exceeds 0.01 micromolars per minute. The output concentrations are changed depending on the type of cell being simulated.

The cells are constrained to be spherical visoelastic objects which move according to Newton's second law as described in (Dallon \& Othmer, 2004; Dallon et al., 2006). For these simulations the cells are constrained to move in a plane and the extracellular cAMP is taken to be uniform in the direction normal to the plane. Depending on the simulations, one of three signals is used to determine if the cells should move. One signal which requires the Tang \& Othmer model is that the cells always move if the dimensionless concentration of $u_{2}$ is greater than 0.004. For typical simulations this means the cells move when they sense a pulse of cAMP. The second is the cells move if the time derivative of the cAMP concentration is greater than 0.1 micromolars per minute (Varnum-Finney et al., 1987). Again the cells will move when they sense a pulse of cAMP. Because cells respond differently to spatial gradients and temporal gradients 
of cAMP (Vicker, 1994; Varnum-Finney et al., 1987; Kriebel et al., 2003), the final rule governing the motion of the cells is that the cells move in a directed manner when the cAMP gradient is above 0.6 micromolars per $\mathrm{cm}$. They move in the direction of the gradient of cAMP for twenty seconds and then they adjust their direction by moving in the direction which is a linear combination of the gradient and their current direction. When cells are close enough, adhesion forces are added to the force equations.

\section{Simulations}

To better understand what affects streaming in the theoretical work, we ran several simulations. The first set of simulations was for a 0.5 by 0.5 centimeter domain with grid space of 50 microns for the computational grid used to calculate the external cAMP concentration. Since the cells were not constrained to the numerical grid, when a cell modifies the cAMP concentration the modification must be transferred to the grid. But the grid spacing was much larger than the cell size, so localization of the cAMP output on the cell membrane could not be modeled. Thus we next looked at simulations on a domain of size 0.8 by $0.8 \mathrm{~mm}$. In these simulations the computational grid spacing was 2 microns which gave several grid points in the area of the cell body (see figure 9 in the appendix) and localization of cAMP output on the cell membrane was possible. There were two basic setups for the simulations. In one there was a periodic output of cAMP at some location in the domain. Typically, this would be a few cells which periodically output cAMP acting as pacemakers for the system. In the other setup there was a constant source of cAMP, designed to mimic the placement of a pipette which creates a spatial gradient of cAMP but not a temporal gradient. For simulations with the periodic output of cAMP the cells either moved according to the threshold of $u_{2}$ if the TO dynamics were used, or moved if the time derivative of cAMP, was above some threshold, when the simple transduction dynamics were used. For simulations of the pipette, unless otherwise stated, the cells moved if the spatial gradient was large enough.

\subsection{Large domain.}

4.1.1. TO dynamics In this set of simulations the domain size is 0.5 by 0.5 centimeter with $5.45 \times 10^{7}$ cells per $\mathrm{ml}$ (recall that the medium is assumed to be about $5.5 \mu \mathrm{m} \mathrm{high}$ ). For the TO simulations of wild type cells with either a pacemaker or a pipette and the $u_{2}$ movement rule, the cells stream and aggregate (see figures 2 and 3 ). The results for the pipette simulation may be an artifact of the TO dynamics. The pipette creates a spatial gradient and when the cAMP concentration is a critical value the cells periodically relay the cAMP signal due to the TO dynamics. The relay provides the temporal gradient which triggers the cell motion. In the experiments with the pipettes the cell's signaling response to the spatial gradient is not clear and it may not relay the signal. At any rate the simulation does highlight that the cAMP signaling is very different when there is a pacemaker versus a pipette. 


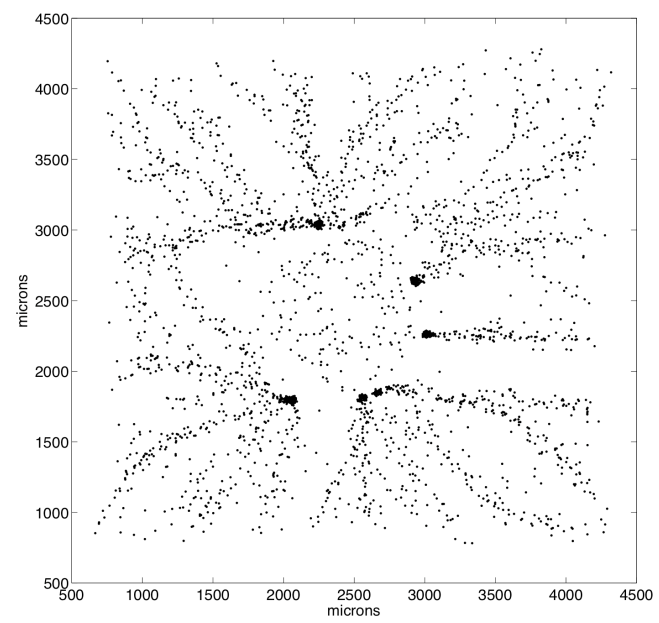

Figure 2: The cell density is shown after 100 minutes of simulation with a pipette in the center (compare with figure 3a). For these simulations the TO dynamics and the $u_{2}$ movement rule are used with 2500 cells each being weighted by a factor of 3 . The simulated pipette outputs about 1 picomole per minute.

Experiments showed that treating Dd aggregations with IPA, which inhibits the activation of ACA (Alvarez-Curto et al., 2007), caused streamless aggregations (Hilgardt et al., 2008). To try and mimic this using the TO dynamics we ran simulations where the weight factor $k$ was higher and lower. This causes the relay signal to be stronger or weaker but it also affects the phosphodiesterases. In the model it corresponds to changing the cell density. The results are shown in figure $3 \mathrm{a}$ ), b) and c). Comparing the results from the normal run in a), one can see that the stronger cAMP signal (higher cell density) in b) caused more well defined streams, and the lower signal strength (low cell density) in c) caused less well defined streams and more smaller aggregations. This is consistent with the experimental results of Hilgardt. To pursue this further we switched to the model with simple dynamics. In the model with simple dynamics we could more easily and selectively manipulate the activation of ACA to mimic the effect of IPA. Additionally the simulations using the simple dynamics gave insight which helps explain the experiments by Kriebel et. al..

4.1.2. Simple dynamics When the simple dynamics are used, the same trend is seen although it is less dramatic. When there is no basal cAMP production and the stimulated production of cAMP is varied, the streaming is less apparent for low levels of cAMP production (see figure 4). When basal production is added to the simulations the results are more striking. In figure 5 the results of simulations where the stimulated production of cAMP is fixed and the basal production rate of cAMP is varied are shown. As the basal rate production of cAMP is increased the streams become less well defined and 


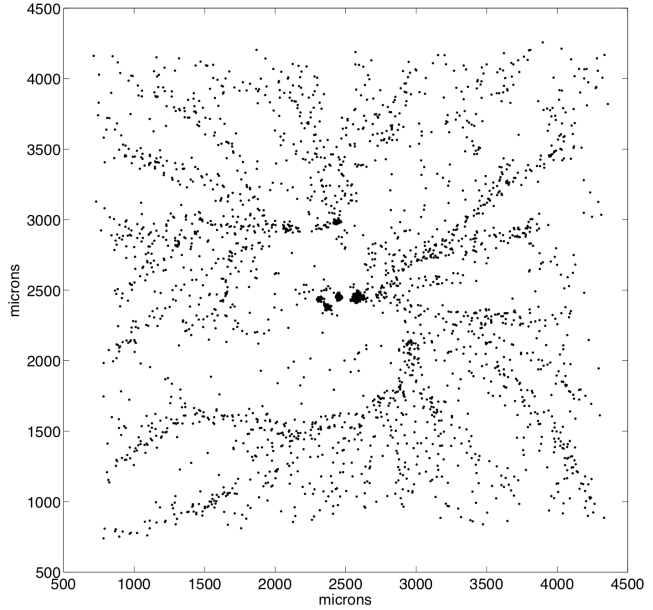

a

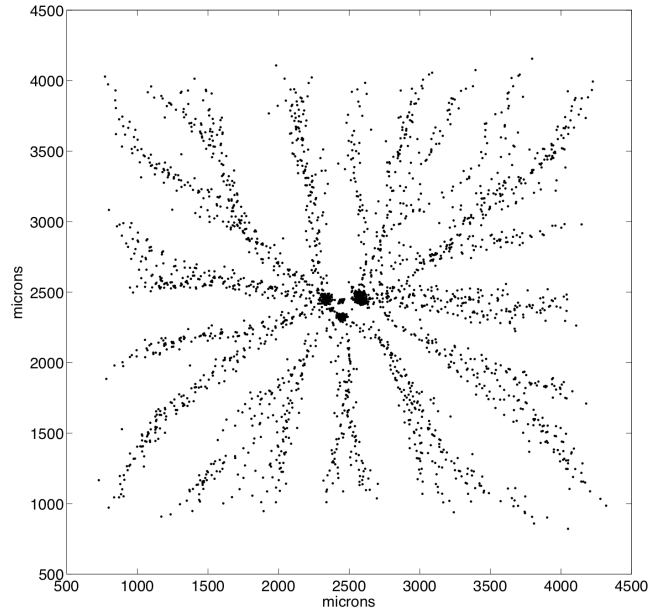

$\mathrm{b}$

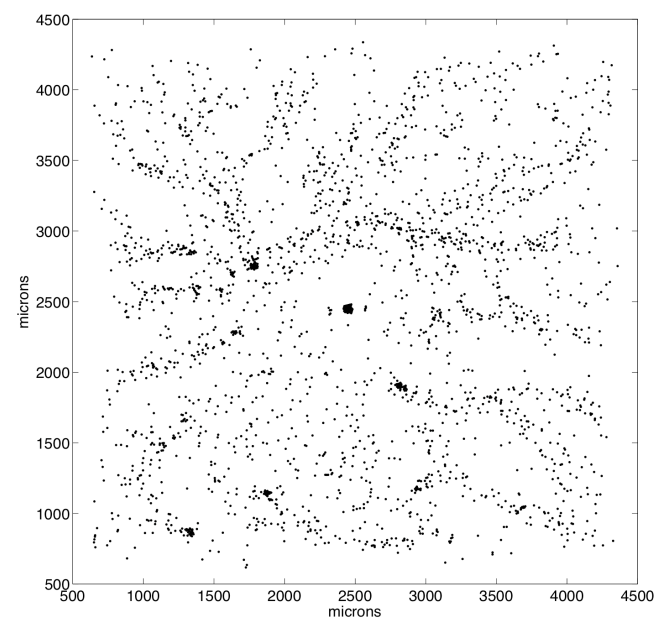

Figure 3: The cell densities are shown for simulations with different weight factors for the signaling response for each cell. Wild type cells are simulated in a) and cells treated with IPA are simulated in c). The cAMP production in b) is two times larger than that in a) and in c) the cAMP production per cell is $2 / 3$ that of the production in a). Note that the simulation with higher cAMP production has more and better formed streams and the simulation with lower production has less stream formation. The cell densities are shown after 100 minutes of simulation where there is a pacemaker in the center region. For these simulations the TO dynamics and the $u_{2}$ movement rule are used with 2500 cells each being weighted by a factor of 3 in a), 6 in b) and 2 in c). 


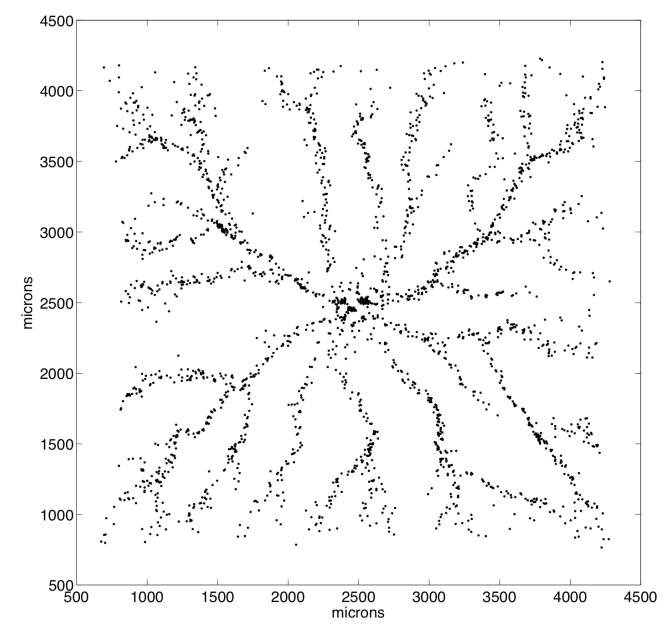

a

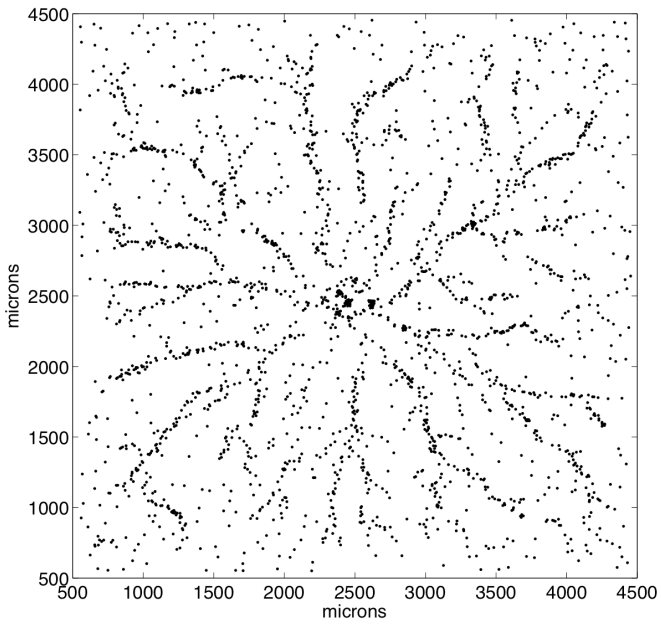

$\mathrm{b}$

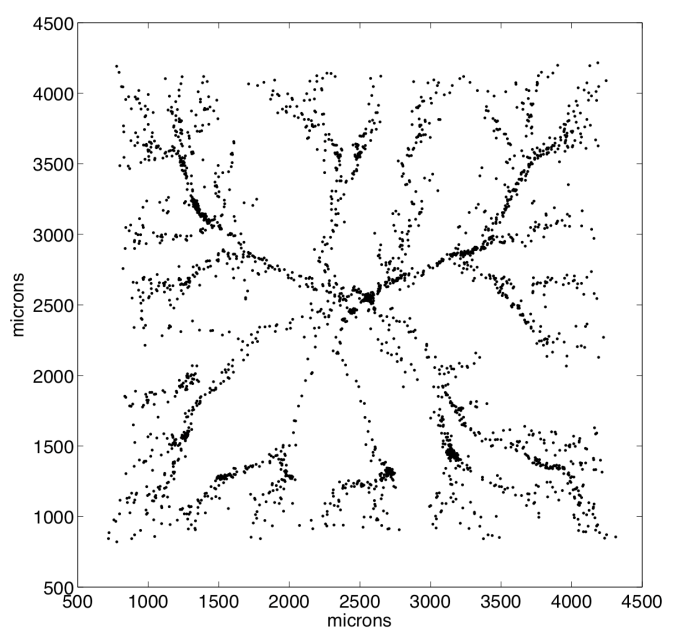

$\mathrm{C}$

Figure 4: The cell densities are shown for simulations with no basal cAMP production and varying strengths of stimulated cAMP production. The cell densities are shown after 60 minutes of simulation where there is a pacemaker in the center region. The cAMP production in b) is less than that in a) which simulates wild type cells. In c) the cAMP production is greater than that in a). Note that the simulation with higher cAMP production has better defined streams and the simulation with lower production has many more thinner streams. For these simulations the simple dynamics are used with the motion determined if the cAMP time derivative is above 0.1 micromolar per minute. The cells signal when the cAMP derivative is greater than 0.01 micromolar per minute and they output $5 \times 10^{6}$ molecules per cell per pulse in a), $5 \times 10^{5}$ in b), and $2 \times 10^{7}$ in c). There are 2500 cells each being weighted by a factor of 3 in all the simulations. 
many mini aggregates form. Some of the simulations are reminiscent of smlA mutant cells (Roisin-Bouffay et al., 2000) where the streams break into smaller aggregations.

It is obvious from these simulations that changes in the production of cAMP can dramatically affect the streaming properties of the system. Recall that the TO dynamics is a system of ordinary differential equations derived from a hypothesized signal transduction pathway for the production of cAMP whereas the simple dynamics simply impose a basal rate of secretion and a fixed form for the stimulated production of cAMP. When switching from the TO dynamics to the simple dynamics the motion rule was also changed from the $u_{2}$ rule to a time derivative rule. Simulations not shown indicate the changes in the motion rule can also affect the streaming patterns, but simulations using the TO dynamics and the time derivative motion rule give the same results. It seems that the contrast between the basal rate and the stimulated production is important in stream formation. When the contrast is greater streams are more readily formed although there is a saturation affect. When the basal production is too high, streams may start to form but seem to breakup.

We can now explain the altered streaming behavior in several of the experiments in a manner consistent with what is know that does not require the cell to localize the cAMP production. The $a c a^{-}$cells, cells which lack adenylyl cyclase ACA, do not respond to the higher cell density regions since the cAMP signal is not dependent on cell density. In simulations where the cAMP signaling of the cells is turned off, the cells which are close enough to the pacemaking region will aggregate into the region uniformly. The $\mathrm{ACA}-\mathrm{C}-\mathrm{YFP} / a \mathrm{ca}^{-}$cells are cells which exhibit high basal rate production of cAMP and a higher production of cAMP when stimulated by G proteins (Kriebel et al., 2003; Parent \& Devreotes, 1996). The high basal rate production of these cells would cause a disruption of the streaming process (see figure 5d). Cells which lack myosin II, myoIIdo not stream and they display severe polarity defects and have an impaired chemotactic response (Wessels et al., 1988). Assuming the lack of polarity would cause the cells to take a more circuitous path, we simulated these cells by increasing the random motion of the cells (see figure 6b). When random motion was added to the simulations with no basal cAMP production the streaming patterns persist but when it was added to simulations where cells produce cAMP at a basal rate of $6.25 \times 10^{4}$ molecules per minute the streaming patterns were not apparent. The $m y o I I^{-}$cells have decreased chemotactic ability. If this is due to more turning and less directed motion, both act to disrupt streaming. That is local regions of higher cell density which are reinforced by chemotaxis towards regions of greater cAMP production causes streaming. Less directed motion and more turning will tend to reduce density of high density regions and increase the density of low density regions thus making the overall density more uniform and result in less streaming. The $c h c^{-}$mutant lacks clathrin and have defects in polarization, turn more frequently, and exhibit poor chemotaxis (Wessels et al., 2000). This mutant also shows defects in streaming. These cells can also be simulated with more random motion. The explanation for the defect in streaming is the same as that for the myoII- cells. Finally, cells treated with CHX move slowly, polarize, turn more frequently (Clotworthy \& Traynor, 2006) and do not stream (Kriebel et al., 2008). This can also be explained by 


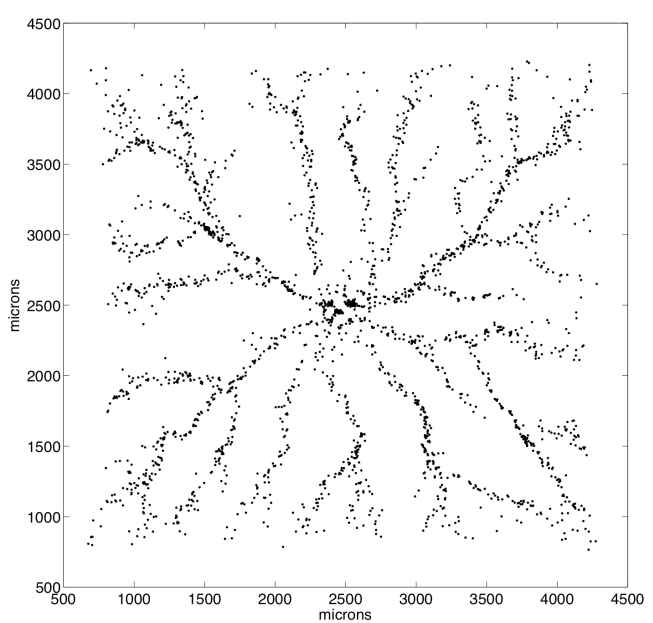

a

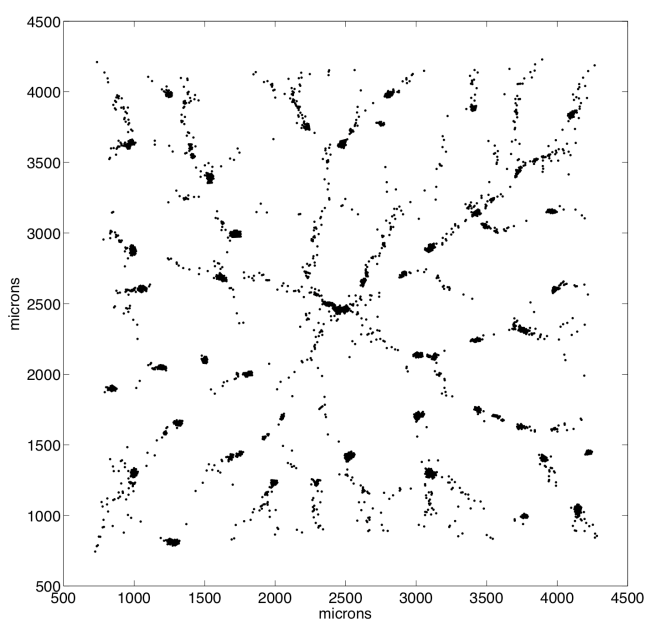

$\mathrm{c}$

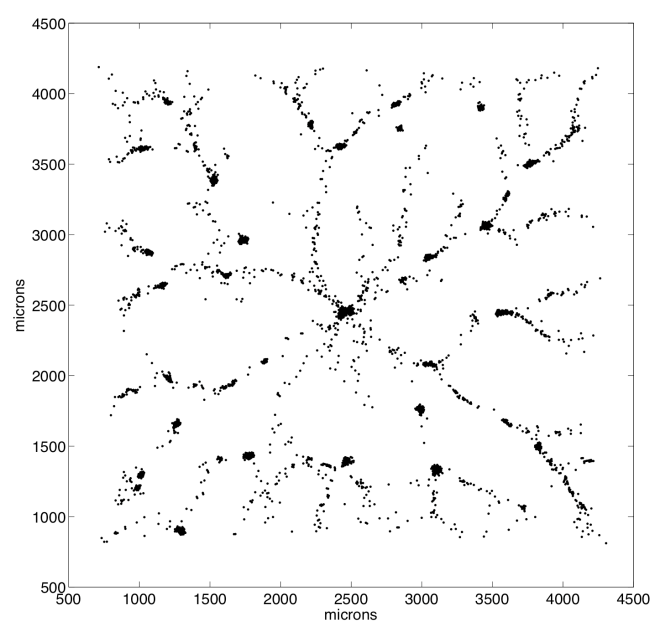

b

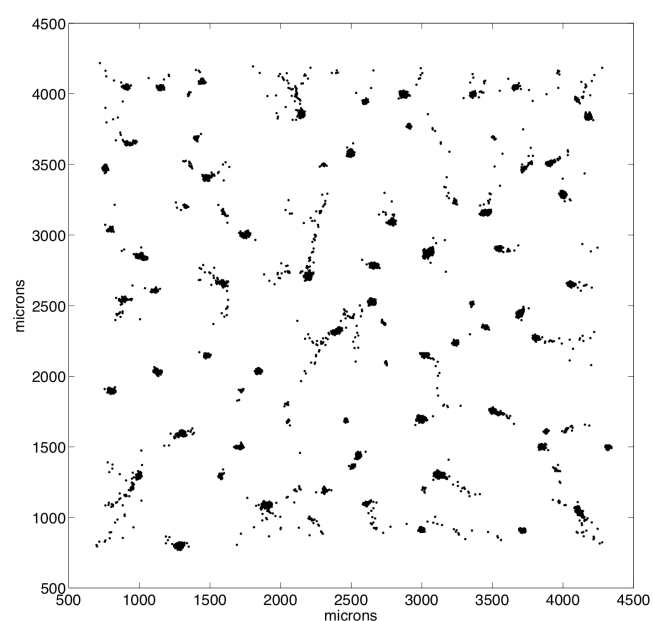

d

Figure 5: The cell density is shown for simulations where the basal production rate of cAMP is varied. In a) there is no basal production (it is the same simulation as shown in figure 4a), in b) the basal rate is $6.25 \times 10^{4}$ molecules per minute, in c) it is $7.81 \times 10^{4}$ molecules per minute, and in d) it is $1.56 \times 10^{5}$ molecules per minute. The cell density is shown after 60 minutes of simulation with a pacemaking region. 


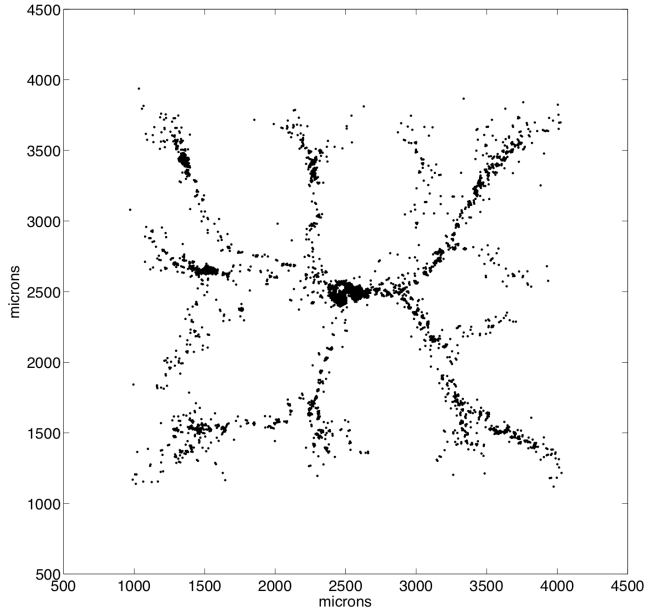

a

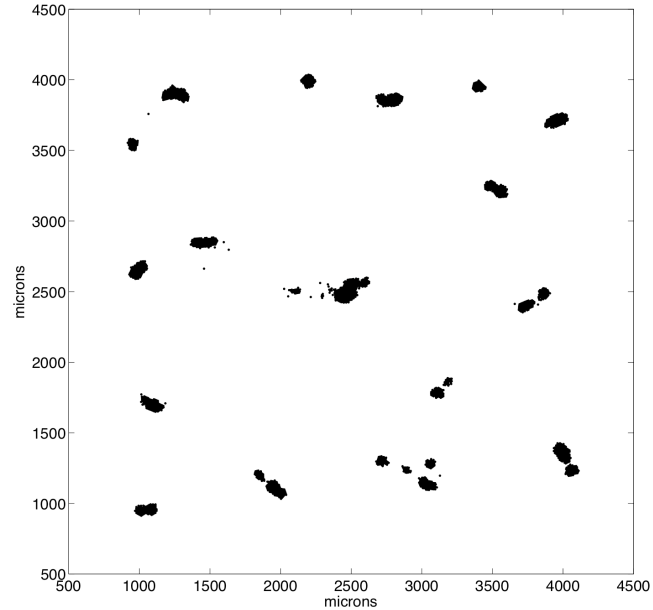

$\mathrm{b}$

Figure 6: The cell density is shown for simulations with random motion where the basal rate of cAMP is varied. In a) there is no basal production of cAMP and in b) the basal rate is $6.25 \times 10^{4}$ molecules per minute. Other than the random motion the simulations are the same as those shown in figure 5 . The force for the random motion is $15 \mathrm{nN}$ which results in cell velocities of about $10 \mu \mathrm{m}$ per minute and when the cells are stimulated to move they have velocities of about $30 \mu \mathrm{m}$ per minute.

the simulation with increased random motion and the smoothing affect of more frequent cell turning on the cell density.

4.2. Smaller domains. In these simulations the domain size is $0.8 \mathrm{~mm}$ by $0.8 \mathrm{~mm}$ with $3.9 \times 10^{7}$ cells per ml. The computational grid is significantly reduced in both space and time, with the separation of the spatial grid being 2 microns and a time step about $1 \times 10^{-5}$ minutes.

4.2.1. Simulations with pacemakers In this set of simulations the aggregation center is due to a pacemaking cell. That is, there is a cell which periodically outputs cAMP which stimulates a wave to propagate through the domain. There are two types of simulations shown. The first is the normal TO simulations and the second is the simulations where the cell outputs the cAMP only at the back half of the cell or the localized ACA cell simulations. For more details about the difference in the simulations, see the appendix. When the TO dynamics are used, the cells clearly stream into higher density regions before aggregating to the pacemaking region (figure $7 \mathrm{a}$ and $\mathrm{c}$ ). Simulations where the output of cAMP is localized to the uropod give similar results (figure $7 \mathrm{~b}$ and $\mathrm{d}$ ). These simulations clearly show that localizing the cAMP production to the uropod of the cell does not affect the streaming nature of the aggregates. 


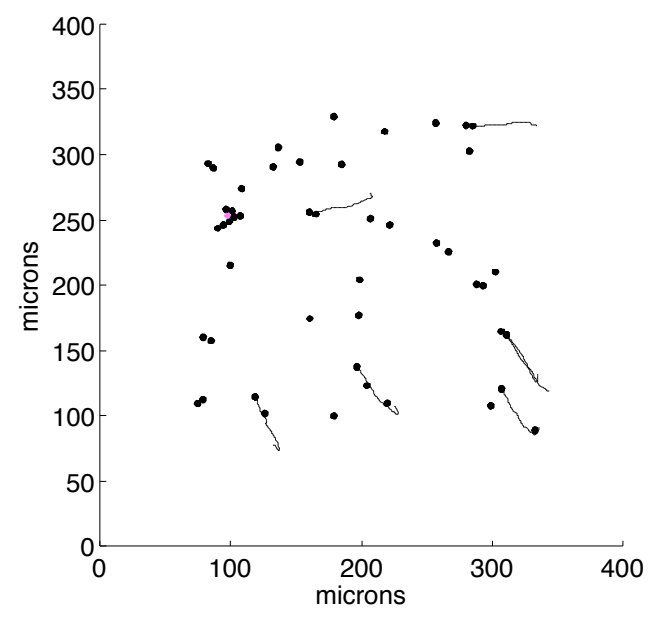

a

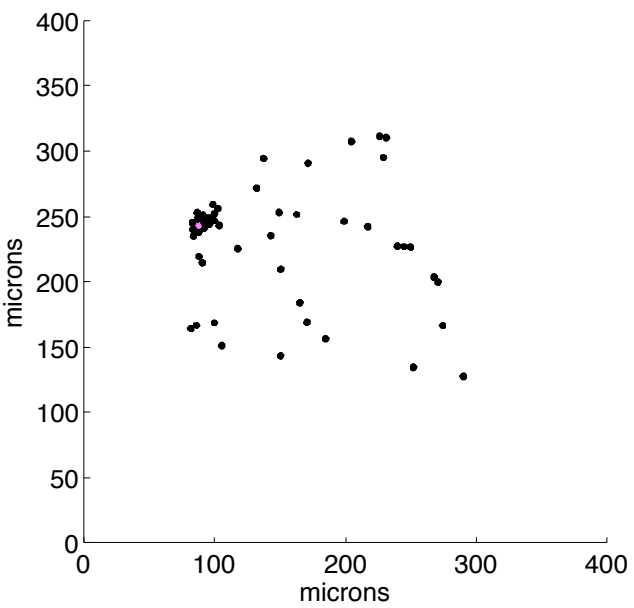

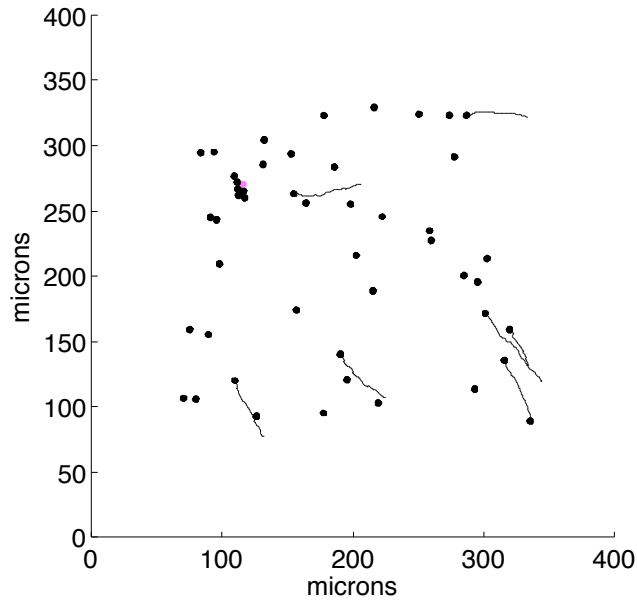

b

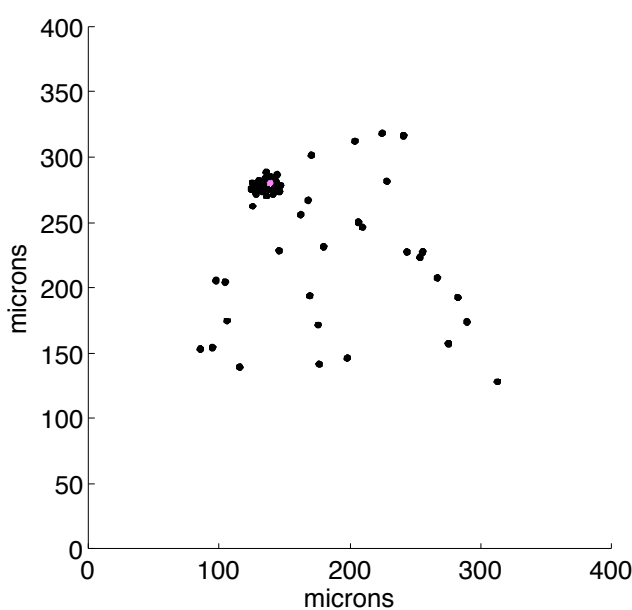

d

Figure 7: Cell positions are shown for simulations of wild type cells which do not localize their cAMP output (a and c) and cells which localize the cAMP output (b and d). In both cases the cells exhibit streaming. In a) and b) the cell density is shown 60 minutes after the simulation has started and the past motion of selected cells is shown by connecting previous cell positions with lines. By continuing the trajectory of many of these cells one can clearly see that the cells are not moving radially into the pacemaking cell. They are moving to regions of higher cell density which are nearby. In all frames the pacemaking cell to which the other cells are aggregating is denoted with a different color. In c) and d) the time shown is 120 minutes. In these simulations the TO dynamics are used with 49 cells randomly placed and each weighted by 4 to give the density stated in the text. 
These simulations indicated that when the cell does not put out cAMP in a uniform manner around its cell membrane, directional information obtained from the cAMP signal by the signalling cell is quickly distorted. That is when the cell outputs cAMP only at its tail end if it waits too long to determine the direction to move it will move towards its uropod and the signal it relayed. When the cell outputs a signal all along its membrane then the directional information is less distorted since the relay increases the cAMP signal in a uniform manner. An asymmetric output of cAMP makes the timing of when a cell chooses a direction to move more critical. A symmetric output of cAMP will not mask the incoming signal as quickly as an asymmetric output.

4.2.2. Pipette Simulations In this final set of simulations the cell aggregation was due to a pipette or a fixed constant source of cAMP as opposed to the periodic source in the previous simulations. In these simulations the simple dynamics were used and the cell motion was determined by the spatial gradients of cAMP. In figure 8, the result of simulations where the basal output of cAMP was varied are shown. In figure $8 \mathrm{a} a c a^{-}$ cells were simulated and there was no streaming. In 8b wildtype cells were simulated and streaming was evident. The other panels $8 \mathrm{c}$ and $\mathrm{d}$ show results where the basal output of cAMP had been increased by a factor of 2 and then 6 , ACA-C-YFP/aca cells should have a behavior between these two simulations. It is clear that although streams started to form, they fragmented. These results look similar to the experimental results where ACA-C-YFP $/ a c a^{-}$cells aggregated into miniclusters and with a stronger pipette source they aggregated to the pipette with severe streaming defects (Kriebel et al., 2003). Under our definition of streaming all the simulations in figure 8 except for panel a, exhibit streaming since the smaller clusters eventually move to the center and join with the main aggregation. The strength of the pipette is important in these simulations. It is the ratio of the pipette strength and the basal production of cAMP which determines if and when the streams fragment. In the computer simulations there is no saturation effect, yet in the biological system saturation would likely occur.

\section{Conclusions}

The apparent contradiction between theory and experimental data can now be explained. The theory predicts that localization of cAMP production at the uropod of the cell is not necessary for streaming. The experimental results are difficult to interpret since several aspects of cell behavior are being modified. By considering the insight gained by the theoretical work the experimental data can be explained in a manner consistent with the theoretical work. It is the other properties of the system which are being altered that are the main cause for the streaming defects and not the localization of the ACA.

The mathematical analysis in previous work indicates streaming is a finite amplitude instability in the model with the TO dynamics. This means that if there is a large enough variation in the density, the system will move away from uniform aggregation and aggregate in a non-uniform manner, for example streaming. Assuming this is true, a consistent explanation of the experiments is possible. There are two main factors which 


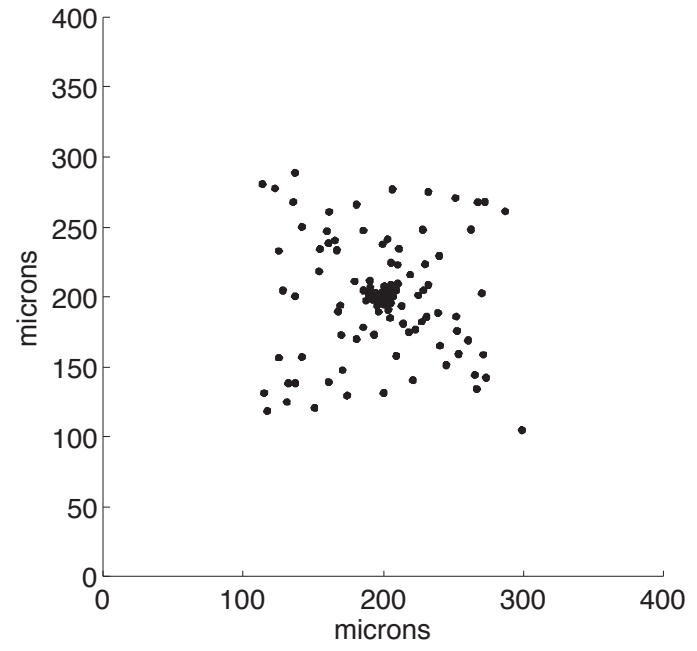

a

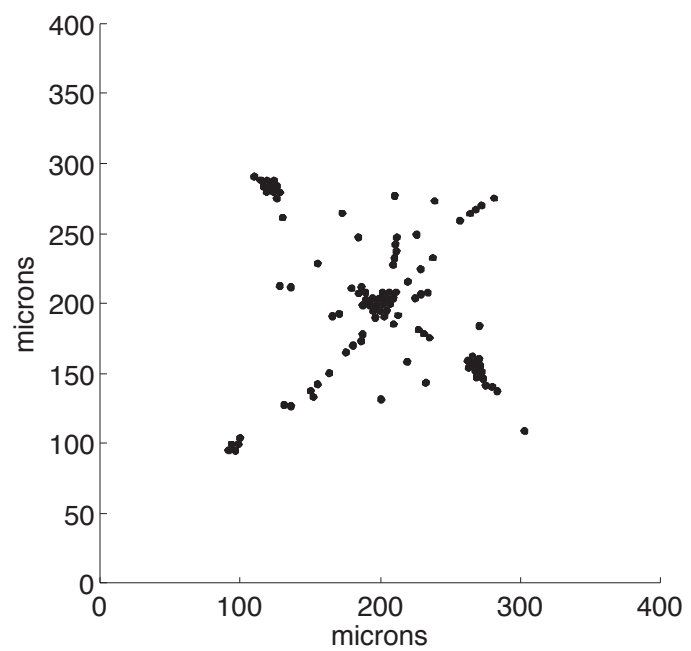

$\mathrm{C}$

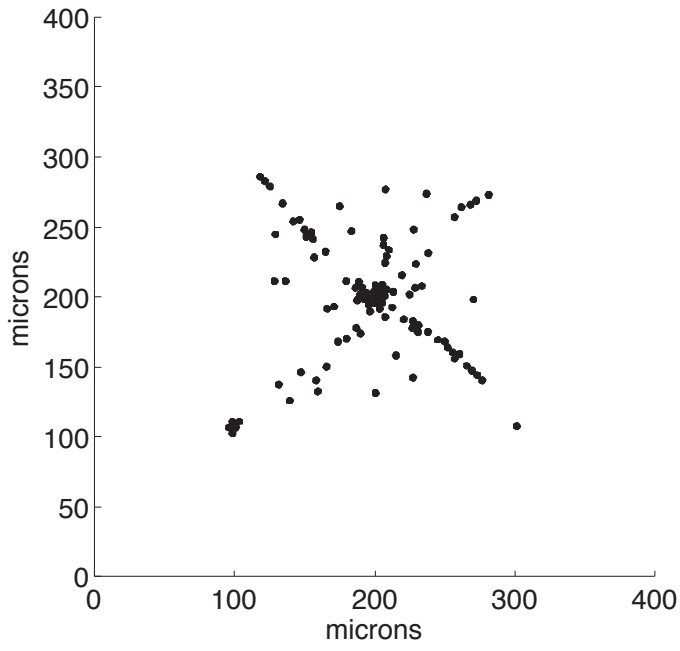

$\mathrm{b}$

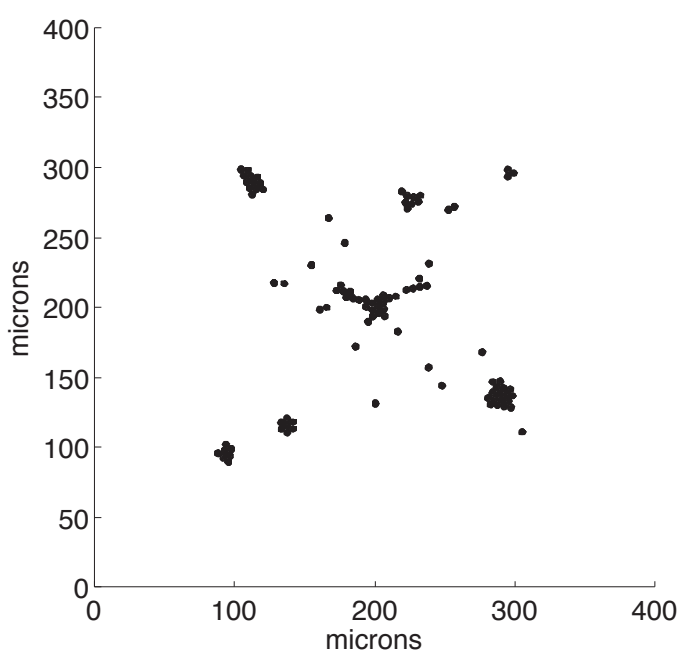

d

Figure 8: Cell positions are shown for simulations with a pipette in the center outputting cAMP at a constant rate. Panel a) represents $a c a^{-}$cells, b) wildtype, and something between c) and d) would represent ACA-C-YFP $/ a c a^{-}$. Notice that in a) no streams form, in b) streams form, in c) and d) the streams start to form but break apart into smaller aggregations. In a) the cells do not produce cAMP, in b) the cells output a basal amount of $4.1 \times 10^{4}$ molecules per minute, in c) $8.3 \times 10^{4}$, and in d) $2.5 \times 10^{5}$. The time is shown 12 minutes after the simulations are started. The simple dynamics are used with 98 cells randomly placed and each weighted by 2 . The pipette releases 0.3 picomoles of cAMP per minute. 
alter the cell density: random cell motion and directed cell motion up cAMP concentration gradient. If the cAMP concentration is higher when cell density is higher, something that occurs when cells relay the cAMP signal, these two factors act in opposition to one another. Random cell motion smooths the cell density by causing higher cell density regions to become less dense and filling in regions of lower density. The directed cell motion up cAMP concentration gradients along with the production of cAMP by the cells causes higher cell density regions to be reinforced by recruiting more cells into those regions. If the initial density or cAMP concentrations are uniform then a uniform, streamless aggregation should occur. On the other hand, if the initial cell density has enough variation, the perturbation away from being uniform has a large enough amplitude, then the higher densities will be reinforced and streaming will occur. Depending on how effective the random motion is at smoothing the cell density and how effective the chemotaxis is at reinforcing the higher density regions will determine if there is uniform aggregation, streaming, or clumping and no aggregation. Of course other factors also influence this, such as cell adhesion which at some scale acts against random motion and helps maintain higher density regions. Yet these two are the essential elements which seem to dominate the system.

Keeping in mind chemotaxis reinforces higher density regions and random motion inhibits higher density regions from forming, one can explain many of the experimental results which affect streaming. When IPA is added, the cell's stimulated production of cAMP is weaker, it is unclear if the basal production of cAMP is altered. The wave of cAMP should have a smaller amplitude and one can assume the gradients are not as strong. Thus the competing force of random motion between waves of cAMP can overcome any reinforcement of higher cell density caused by the chemotaxis. Streams may start to form with each wave but the random motion will disperse the cells sufficiently that the critical density which allows the stable streams to form is not attained. In the experiments with $\mathrm{ACA}-\mathrm{C}-\mathrm{YFP} / \mathrm{aca}^{-}$cells the rate of basal production of cAMP is greatly increased. It is true that the stimulated production of cAMP is also increased but there is likely a saturation effect. Thus the ACA-C-YFP $/ a c a^{-}$do not stream because the basal rate is high enough that the cells lose the main aggregation signal in the strong cAMP gradients. The motion of the cells is more random in nature because the chemotactic signal does not cause enough coordinated motion to aggregate the cells to one center. The cells clump in several miniaggregates. If the strength of the main aggregation signal is increased enough, the cells will aggregate towards it but still do not stream since the background cAMP signal is too strong for them to find local regions of higher density. The $a c a^{-}$cells are lacking the relay and thus the chemotaxis does not reinforce higher density regions and there is no streaming. The $\mathrm{myoII}^{-}$cells have an impaired chemotactic response, as do $\mathrm{chc}^{-}$cells and CHX treated cells. The last two also have increased random turning, thus streaming should be impaired in all three cases. There are not enough properties known about the $c c^{-}$cells to explain why they have defective streaming.

In all of these experiments, the localization of ACA to the uropod does not seem to be a factor in the initiation of stream formation. It is the alterations in the cAMP 
signal the cell senses, the chemotactic ability of the cells, or the cell motion that affect the streaming. Localization of ACA may be important in forming cell-cell contact, reinforcing or stabilizing streams once they are formed. For example it could affect the adhesive properties of the cell membrane. The localization may be a result of localization of some part of the signal transduction components. Perhaps the localization allows more receptors for the cAMP signal to be the leading edge of the cell. Our work does not address any of these issues. Clearly more thought and experimentation are necessary to fully understand what features of the system cause streaming and why ACA localizes at the cell uropod.

\section{References}

E. Alvarez-Curto, K. E. Weening, \& P. Schaap. (2007) Pharmacological profiling of the Dictyostelium adenylate cyclases ACA, ACB and ACG. Biochem. J., 401, 309-316.

M. Clotworthy, \& D. Traynor. (2006) On the effects of cycloheximide on cell motility and polarisation in Dictyostelium discoideum. BMC Cell Biol.

M. H. Cohen, D. J. Drage, \& A. Robertson. (1975) Iontophoresis of cyclic AMP. Biophys. J., 15, 753-763.

J. C. Dallon. (2000) Numerical Aspects of Discrete and Continuum Hybrid Models in Cell Biology. Applied Numerical Mathematics, 32, 137-159.

J. C. Dallon, \& H. G. Othmer. (1997) A discrete cell model with adaptive signalling for aggregation of Dictyostelium discoideum. Phil. Trans. R. Soc. Lond. B, 352(1357), 391-417.

J. C. Dallon, \& H. G. Othmer. (1998) A Continuum Analysis of the Chemotactic Signal Seen by Dictyostelium discoideum. J. Theor. Biol., 194, 461-483.

J. C. Dallon, \& H. G. Othmer. (2004) How cellular movement determines the collective force generated by the Dictyostelium discoideum slug. J. Theor. Biol., 231, 203-222.

J. C. Dallon, W. Jang, \& R. H. Gomer. (2006) Mathematically modeling the effects of counting factor (CF) in Dictyostelium discoideum. Math. Med. Biol., 23, 45-62.

P. N. Devreotes, \& T. L. Steck. (1979) Cyclic 3',5' AMP relay in Dictyostelium discoideum II. Requirements for the Initiation and Termination of the Response. $J$. Cell Biol., 80, 300-309.

G. Gerisch, \& U. Wick. (1975) Intracellular oscillations and release of cyclic AMP from Dictyostelium cells. Biochem. Biophys. Res. Commun., 65, 364-370.

C. Hilgardt, J. Cejkova, M. J. B. Hauser, \& H. Sevcikova. (2008) Streamless aggregation of Dictyostelium in the presence of isopropylidenadenosin. Biophysical Chemistry, 132, 9-17.

T. Hoefer, J. A. Sherratt, \& P. K. Maini. (1995) Dictyostelium discoideum: cellular selforganization in an excitable biological medium. Proc. R. Soc. Lond. Biol., 249-257.

T. Hofer, \& P. K. Maini. (1997) Streaming instability of slime mold amoebae: An analytical model. Physical Review E, 56(2), 2074-2080.

R. H. Kessin. (2001) Dictyostelium Evolution, cell biology, and the development of multicellularity Developmental and cell biology series Cambridge University Press. 
P. W. Kriebel, V. A. Barr, \& C. A. Parent. (2003) Adenylyl cyclase localization regulates streaming during chemotaxis. Cell, 112, 549-560.

P. W. Kriebel, V. A. Barr, E. C. Rericha, G. Zhang, \& C. A. Parent. (2008) Collective cell migration requires vesicular trafficking for chemoattractant delivery at the trailing edge. J. Cell Biol., 183, 949-961.

H. Levine, I. Aronson, L. Tsimring, \& T. V. Truong. (1996) Positive genetic feedback governs cAMP spiral wave formation in Dictyostelium. Proc. Nat. Acad. Sci. USA, 93, 6382-6386.

W. F. Loomis. (1975) Dictyostelium discoideum: A Developemental System New York: Academic Press.

S. MacKay. (1978) Computer simulation of aggregation in Dictyostelium discoideum. J. Cell Sci., 33, 1-16.

H. G. Othmer, \& P. Schaap. (1998) Oscillatory cAMP Signaling in the Development of Dictyostelium discoideum. Comments on Theor. Biology, 5, 175-282.

E. Palsson, \& H. G. Othmer. (2000) A Model for Individual and Collective Cell Movement in Dictyostelium discoideum. Proc. Nat. Acad. Sci. USA, 97, 10448-10453.

C. A. Parent, \& P. N. Devreotes. (1996) Constitutively Active Adenylyl Cyclase Mutant Requires Neither G Proteins nor Cytosolic Regulators. J. Biol. Chem., 271(31), 18333-18336.

H. Parnas, \& L. A. Segel. (1977) Computer evidence concerning the chemotactic signal in Dictyostelium discoideum. J. Cell Sci., 25, 191-204.

C. S. Peskin. (1977) Numerical analysis of blood flow in the heart. J. Comp. Phys., 25, 220-252.

T. Höfer, J. A. Sherratt, \& P. K. Maini. (1995) Dictyostelium discoideum: cellular selforganization in an excitable biological medium. Proc. R. Soc. Lond. B, 259(1356), $249-257$.

C. Roisin-Bouffay, W. Jang, D. R. Caprette, \& R. H. Gomer. (2000) A Precise Group Size in Dictyostelium is Generated by a Cell-Counting Factor Modulating Cell-Cell Adhesion. Molecular Cell, 6(October), 953-959.

W. Roos, V. Nanjundiah, D. Malchow, \& G. Gerisch. (1975) Amplification of cyclic AMP signals in aggregating cells of Dictyostelium discoideum. 53, 139-142.

N. Savill, \& P. Hogeweg. (1997) Modelling morphogenesis: from single cells to crawling slugs. J. Theor. Biol., 184, 229-235.

Y. Tang, \& H. G. Othmer. (1995) Excitation, oscillations and wave propagation in a G-protein based model of signal transduction in Dictyostelium discoideum. Phil. Trans. Roy. Soc. (Lon.), B349, 179-195.

B. Varnum-Finney, K. Edwards, E. Voss, \& D. Soll. (1987) Amebae of Dictyostelium discoideum respond to an increasing temporal gradient of the chemoattractant cAMP with a reduced frequency of turning: evidence for a temporal mechanism in ameboid chemotaxis. Cell Motil. Cytoskel., 8(1), 7-17.

B. Vasiev, F. Siegert, \& C. J. Weijer. (1997) A hydrodynamic model for Dictostelium discoideum mound formation. J. Theor. Biol., 184, 441-450.

B. N. Vasiev, P. Hogeweg, \& A. V. Panfilov. (1994) Simulation of Dictyostelium discoideum Aggregation via Reaction-Diffusion Model. Phys. Rev. Letts., 73(23)(December), 3173-3176. 
M. G. Vicker. (1994) The regulation of chemotaxis and chemokinesis in Dictyostelium amoebae by temporal signals and spatial gradients of cyclic AMP. J. Cell Sci., 107(Pt 2), 659-667.

D. Wessels, J. Reynolds, O. Johnson, E. Voss, R. Burns, K. Daniels, E. Garrard, T. J. O'Halloran, \& D. Soll. (2000) Clathrin plays a novel role in the regulation of cell polarity, pseudopod formation, uropod stability and motility in Dictyostelium. $J$. Cell Sci., 113(1), 21-36.

D. Wessels, D. Soll, D. Knecht, W. Loomis, A. D. Lozanne, \& J. Spudich. (1988) Cell Motility and Chemotaxis in Dictyostelium. Dev. Bio., 128(1), 164-177.

\section{Appendix}

6.1. Small domain TO dynamics. In the simulations where the domain size is $0.8 \mathrm{~mm}$ by $0.8 \mathrm{~mm}$ with $3.9 \times 10^{7}$ cells per $\mathrm{ml}$, the way the cells interact with the extracellular cAMP changes. In all the simulations the cell senses the different properties of the extracellular cAMP by interpolating to the center location of the cell as described in previous work (Dallon, 2000). The manner in which the cell adds its contribution to the extracellular cAMP differs since there are several numerical grid points contained in the area of a cell for these simulations, which was not the case in the larger domain simulations. For the nonlocalized simulations shown in figure 7 , the cell's output is distributed to the numerical grid by using the discretized $\delta$ function given by

$$
\begin{gathered}
\delta_{r}(x, y)=\delta_{r}(x) \delta_{r}(y) \\
\delta_{r}(x)= \begin{cases}\frac{1}{2 r}\left(1+\cos \left(\frac{\pi x}{r}\right)\right) & |x| \leq r \\
0 & |x| \geq r\end{cases}
\end{gathered}
$$

where $r$ is the cell radius (Peskin, 1977) in equations 6. Figure 9a shows the grid points which would be affected by a typical cell.

For the localized simulation, figure $9 \mathrm{~b}$ shows the grid points which would be affected by a typical cell depending on the direction the cell was moving. To determine which numerical grid points get a contribution from the cell, the closest of the eight directions shown in the figure to the direction the cell is moving is determined. The support of $\delta_{r}(x, y)$ is the portion of the square darkened in the figure. This localizes the output to the uropod of the cell. Additionally the argument of $\delta_{r}(x)$ is changed to $\delta_{r}(r-x)$ and the function is normalized over the new domain. This causes the maximum output to occur near the cell membrane and the normalization makes the output have the correct magnitude. The only other change is that the sign of the term multiplying the $\delta$ function in equation 6 ,

$$
\left(s r\left(w_{4}^{i}\right)-\gamma_{7} \frac{w_{5}(\mathbf{x})}{w_{5}(\mathbf{x})+\gamma_{6}}\right)
$$

is set to zero if it is negative, i.e. the output of a cell cannot be negative. This means that the membrane phosphodiesterase only degrades the cAMP which is produced by 

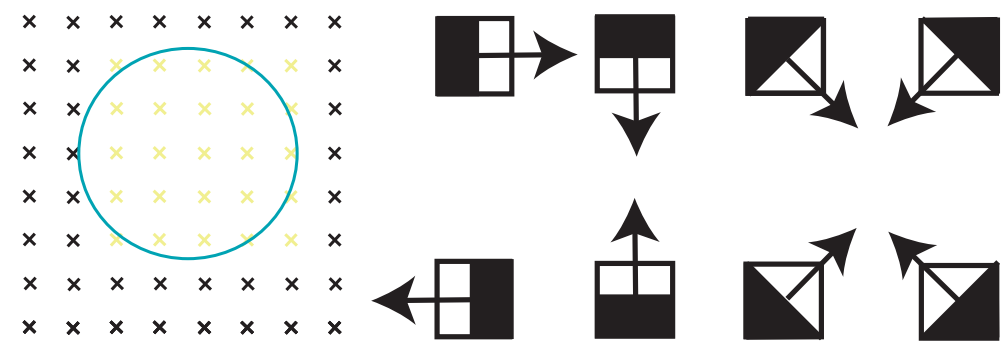

a

$\mathrm{b}$

Figure 9: In a) the numerical grid points, denoted by X, which would be affected by the discretized $\delta$ function are shown in yellow. In b) squares indicate the collection of red grid points in a). Depending on the cells direction one of the eight cases is used for the localized simulations. The support of the discretized $\delta$ function is shown in black for these cells.

the cell and the cell cannot act as a sink. This was done for numerical convenience. Comparisons of the nonlocalized cells which can act as sinks and cells which cannot act as sinks have been performed and show little difference. 